Research Article

\title{
Simulation and Analysis of GaN Wafer Bowing on Sapphire Substrate
}

\author{
Wang Bin, ${ }^{1}$ Qu Yu-xuan, ${ }^{2}$ Hu Shi-gang, ${ }^{3}$ Tang Zhi-jun, ${ }^{3}$ Li Jin, ${ }^{3}$ and Hu Ying-lu ${ }^{4}$ \\ ${ }^{1}$ The Center of Coordination and Support of State Administration of Science, Technology and Industry for National Defence, \\ Beijing 100081, China \\ ${ }^{2}$ Advanced Technology Generalization Institute of CNGC, Beijing 100089, China \\ ${ }^{3}$ School of Information and Electrical Engineering, Hunan University of Science and Technology, Xiangtan 411201, China \\ ${ }^{4}$ The 41st Institute of China Electronics Technology Group Corporation, Qingdao 266555, China
}

Correspondence should be addressed to Hu Shi-gang; hsg99528@126.com

Received 17 June 2013; Accepted 14 August 2013

Academic Editor: Jianhua Hao

Copyright (c) 2013 Wang Bin et al. This is an open access article distributed under the Creative Commons Attribution License, which permits unrestricted use, distribution, and reproduction in any medium, provided the original work is properly cited.

During the process of heteroepitaxial growth, if the lattice constant of the growing film differs from that of the substrate, the wafer surface bows, regardless of whether the lattice mismatch occurs or not. As the growth in large-scale wafers speeds up, bowing effects are becoming more and more important. Wafer bowing has a direct impact on the yield in modern mass-production compound semiconductor industries. By using finite element analysis software, the bowing deformation of the GaN wafer on sapphire substrate can be studied. This paper summarizes the causes of bowing deformation, builds the mathematical model, and deduces the relation equation of the wafer bowing. The results show that epitaxial wafer bowing has a linear relationship with the square of the diameter of the substrate but has little relationship with the thickness of the substrate. Moreover, the relation equation of the wafer bowing is also simplified finally.

\section{Introduction}

Using MOCVD (metal-organic chemical vapor deposition) to analyze the growth of GaN-base photoelectric devices on the sapphire substrate is a common method in the semiconductor lighting industry [1-5]. In recent years, along with the unceasing enhancement of epitaxy technology and related technologies, larger sapphire substrates are needed. The optimization and improvement of MOCVD reaction chamber structure with large-scale epitaxial wafers have been studied by many domestic researchers [6-10]. Li et al. [11, 12] optimized high-frequency heating graphite base groove structure with eight-inch and twelve-inch substrates. Yinglu et al. [13] studied the heating modulate curve of radiation heating MOCVD and proposed the design principles of the outer heater. To improve the production efficiency, some great MOCVD manufacturers also focus on how to improve and increase the MOCVD cavity and the sapphire substrate size in foreign countries. At present, two-inch and four-inch epitaxial wafers are frequently used, so manufacturers who can produce six-inch $\mathrm{SiC}$ and $\mathrm{Si}$ substrate epitaxial wafers with high quality in foreign countries would sell chips abroad $[14,15]$.

With the growth of the III-nitrides, sapphire becomes the most extensively used substrate material [16]. Crystals of sapphire with good quality and low price can be easily got. Besides, sapphire is stable at high temperature and the growth technology of nitrides on sapphire is now fairly mature. However, the problem of wafer bowing, which results from the difference in thermal expansion coefficient between $\mathrm{GaN}$ epitaxial layer and sapphire, has become much more serious in larger-diameter wafers. It will deteriorate the contact between the substrates and the equipment stages or the subsectors during device process, which will result in degradation in the device uniformity or failures in lithography.

By using finite element simulation software, the relationship between the maximum wafer bowing and sapphire substrate size under characteristic temperature is studied in 
the case of the growth of $\mathrm{GaN}$ in the sapphire substrate, and the wafer bowing relation equation is also deduced. The research work in this paper plays a foreshadowing role for later research sapphire epitaxial growth and MOCVD reaction chamber structure improvement.

\section{Bowing Causes and Mathematical Model}

2.1. Bowing Causes. The MOCVD growth process can be divided into four phases: a reactant input phase, a reactant mixing phase, an immediate boundary layer phase above the substrate, and the growth phase on the substrate surface itself. Growth complications that can occur in these phases include gas phase reactions during reactant mixing, reactant diffusion and/or pyrolysis in the boundary layer above the substrate, and thermodynamic or kinetic rejection of species from the substrate. The worst effects can be reduced or eliminated by providing appropriate equipment design and process conditions [17].

As shown in Figure 1, MOCVD growth system is composed of four parts including gas handle system, reactor, computer control, and vacuum and exhaust system.

The stress in the films often results from epitaxy in the growth processes and from thermal expansion coefficient (TEC) mismatch in the postgrowth processes of both metalorganic chemical vapor deposition and hydride vapor phase epitaxy. For example, the stress typically observed is dominated by thermal stress in the post-growth processes, which is usually considered to bring defects or damage to the films, such as, dislocation, buckling, and cracking. Cracks normally extend to the principal tensile stresses within the thin brittle layers and interact with the interfaces of $\mathrm{GaN} /$ sapphire. Due to the mismatch of TECs between the GaN film and sapphire, wafer bowing will occur when the GaN film is mechanically constrained by the sapphire after cooling. Thermal stress, wafer bending, and cracking are the main drawbacks that hamper the production of large-area GaN substrates and the application of GaN films. Wafer bowing and stress concentration significantly influence not only the devices' mechanical performance but also their optical, electrical, and magnetic properties. Therefore, reducing wafer bowing and stress concentration in GaN films is very important. To the best of our knowledge, there are no feasible methods for reducing $\mathrm{GaN}$ wafer bowing and few papers on wafer bowing have been published.

When GaN is grown on the sapphire substrate using MOCVD, bowing will happen in epitaxial wafers. There are three major factors for the wafer bowing. One is the temperature difference between the upper and lower interfaces of epitaxial wafers. The lower surface temperature is higher than that of the upper surface, so that the expansion of the lower surface can be larger than that of the upper surface, causing the epitaxial wafers concave. Another is the lattice mismatch between sapphire substrate and epitaxial material, which makes the sapphire suffer from tensile and compressive stress and produces concave or convex bowing. The third is thermal mismatch between epitaxial material and

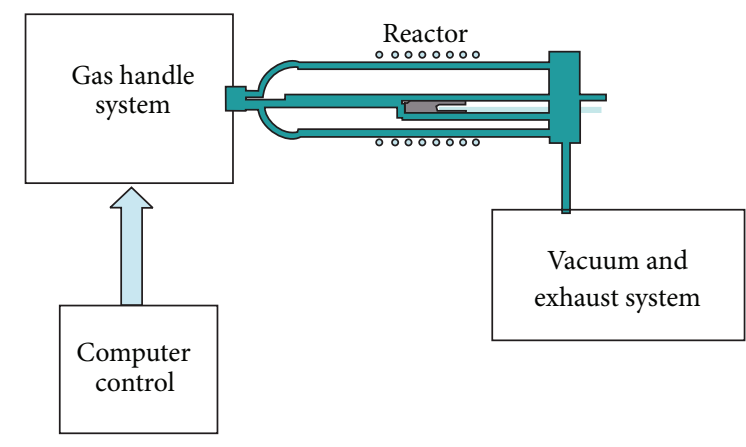

FIGURE 1: Schematic representation of MOCVD growth system.

the sapphire substrate, which occurs mainly in the case of a sudden jump or a sudden drop of the outside temperature.

2.2. Mathematical Model. This paper mainly studies the maximum bowing situation in the growth process. Since the epitaxial layer is much thinner than the sapphire substrate, considering the changes of temperature difference due to lattice mismatch and epitaxial layer heat transfer, the upper surface of epitaxial wafer is approximated to the thermal radiation condition of GaN. Since the condition is steady state, the thermal mismatch between epitaxial materials and sapphire substrate can be ignored. Therefore, the bowing of GaN epitaxial wafer with sapphire substrate is mainly due to the temperature difference between the upper and lower interfaces in this paper.

The solid lattice conforms to the Grüneisen lattice state equation generally:

$$
P=-\frac{d E_{0}}{d V}+\gamma \frac{E_{L}}{V}
$$

The first item is known as static pressure, and the second item is called thermal pressure, where $E_{0}$ is a function of the crystal volume $V, E_{L}$ is the average lattice vibration at the temperature $T$, and $\gamma$ is called Grüneisen constant

$$
\begin{gathered}
E_{L}=\sum_{j}\left(\frac{1}{2} \hbar \omega_{j}+\frac{\hbar \omega_{j}}{e^{\hbar \omega_{j} / k_{B} T}-1}\right), \\
\gamma=-\frac{d \ln \omega}{d \ln V} .
\end{gathered}
$$

Solid thermal expansion refers to the change of volume along with temperature in the case without pressure. Making $P=0$, we can get

$$
\frac{d E_{0}}{d V}=\gamma \frac{E_{L}}{V}
$$

Because the change of volume is not big during general thermal expansion, $d E_{0} / d V$ can be expanded near the lattice volume $V_{0}$, and only one degree term of $\Delta V=V-V_{0}$ is retained, we can get

$$
\frac{d E_{0}}{d V}=\left(\frac{d E_{0}}{d V}\right)_{V_{0}}+\left(\frac{d^{2} E_{0}}{d V^{2}}\right)_{V_{0}} \Delta V
$$


Considering that the first term is equal to 0 and the definition of bulk modulus, then

$$
K_{0}=V_{0}\left(\frac{d^{2} E_{0}}{d V^{2}}\right) V_{0}, \quad \frac{d E_{0}}{d V}=K_{0} \frac{\Delta V}{V_{0}} .
$$

Substituting formula (3), we can get

$$
\frac{\Delta V}{V_{0}}=\gamma \frac{E_{L}}{K_{0} V} .
$$

Solving the derivative of the formula above with respect to temperature and using the definition of heat capacity $C_{V}=$ $\partial E_{L} / \partial T$, the volumetric thermal expansion coefficient is obtained:

$$
\alpha=\gamma \frac{C_{V}}{K_{0} V} .
$$

For the solid material, the Grüneisen constants are between 1 and 2 generally. Assuming that the entire heat capacity of the graphite disc is the same, as well as the small temperature difference between the upper and lower surfaces of the epitaxial wafer, the thermal expansion of epitaxial wafer can be approximated as a linear expansion.

Without affecting the main results, the temperature of the lower surface of epitaxial wafer is set to the constant temperature $1220 \mathrm{~K}$, which is close to the actual temperature. Assuming that the sapphire substrate is isotropic in a plane, its deformation meets the equation of thermal expansion:

$$
L=\alpha L_{0}\left(T-T_{0}\right)
$$

$L_{0}$ and $L$ are the amounts before and after the expansion, respectively, $\alpha$ is the coefficient of thermal expansion, and $T_{0}$ is the reference temperature.

Epitaxial wafer deformation models and geometrical parameter identification are shown in Figure 2. $R$ is sapphire substrate radius size, $d$ is the thickness of the epitaxial wafer, and $B$ is the highest bowing degree of epitaxial wafer.

It is assumed that the bowing of the epitaxial wafer is caused by the different degrees of thermal expansion due to the average temperature difference between the upper and lower surfaces of the sapphire. As shown in Figure 3, we have set thermal expansion coefficient of sapphire for $\alpha$, average temperature of the upper surface for $T_{1}$, the average temperature of the lower surface for $T_{2}$, reference temperature $T_{0}$, radian formed by sapphire bowing for $\theta$, and cambered radius of the upper surface for $r$.

By the law of thermal expansion and the geometric relationship, it can be got that

$$
\begin{gathered}
\alpha R\left(T_{1}-T_{0}\right)=\theta r, \\
\alpha R\left(T_{2}-T_{0}\right)=\theta(r+d) .
\end{gathered}
$$

Deformation is quite slimly relative to the substrate size, so the angle $\theta$ is very small:

$$
\frac{1}{2} \theta \approx \frac{1}{2} \tan \theta \approx \frac{B}{R}
$$

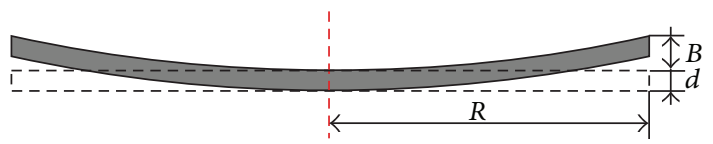

FIGURE 2: Epitaxial wafer model.

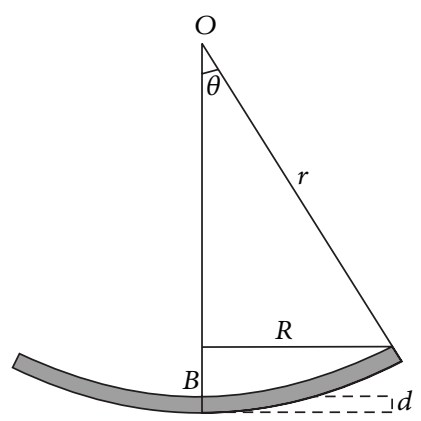

FIGURE 3: Epitaxial wafer bowing deformation schematic diagram.

Let $\Delta T=T_{2}-T_{1}$; then it can be derived that

$$
B \approx \frac{1}{2} \alpha R^{2} \frac{\Delta T}{d} .
$$

As can be seen from the above derivation, the maximum bowing is proportional to the square of the epitaxial wafer substrate radius, and the temperature difference between the upper and lower surfaces of epitaxial wafers is inversely proportional to the thickness of the epitaxial wafers.

\section{Simulation Results and Analysis}

3.1. Influence of Sapphire Size on the Deformation. To observe the bowing with the same thickness of $500 \mathrm{~nm}$, the diameters of the sapphire substrate $2 R$ with two inches, four inches, six inches, and eight inches were compared. The simulation results are shown in Figure 4.

With the increase of size, the degree of bowing is also growing. When the diameter of the substrate is eight inches, its bowing has reached nearly $500 \mathrm{~nm}$, similar to the thickness of the epitaxial wafers of sapphire substrate, which can lead to the rejection of disk accident. It is obviously one of the main problems needed to be solved when large-area sapphire substrate is epitaxial. As shown in Figure 5, the bowing amount and the square of sapphire diameter (radius) are seen in a roughly linear relationship; that is to say, $B \approx A R^{2}$, where $A$ is a constant.

3.2. Influence of the Thickness of Sapphire Size on the Deformation. Under the same heating condition, we researched the bowing degree of several four-inch substrates of which the thickness is $300 \mathrm{~nm}, 400 \mathrm{~nm}, 500 \mathrm{~nm}$, and $600 \mathrm{~nm}$, respectively. It can be seen in Figure 6, in the case of different thicknesses, the degree of the bowing only changes by a few nanometers, which can be neglected, that is, the thickness has less influence on the bowing.

There are a few differences between this conclusion and the above theoretical result. It is considered that the temperature difference between the upper and lower substrate 


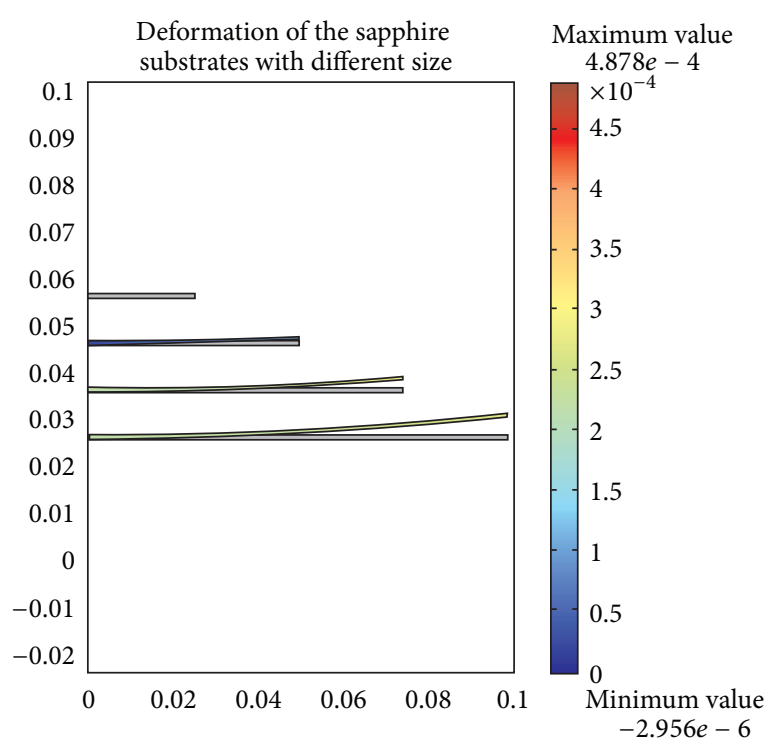

(a)

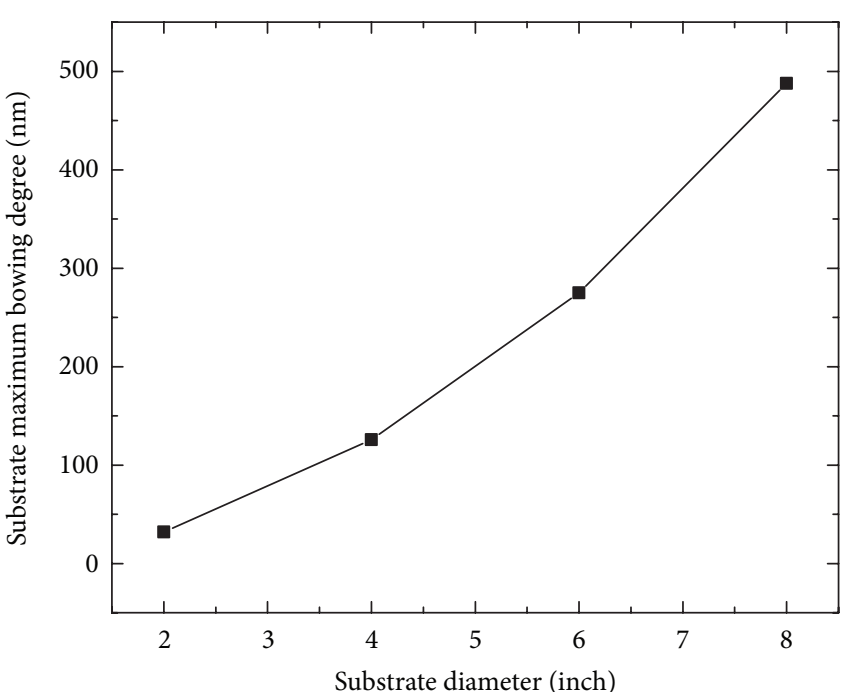

(b)

FIGURE 4: Simulation results of bowing amount of different size substrate (a) deformation of sapphire substrate with different sizes (10 times effect), (b) relation curve of maximum amount of bowing and substrate diameter.

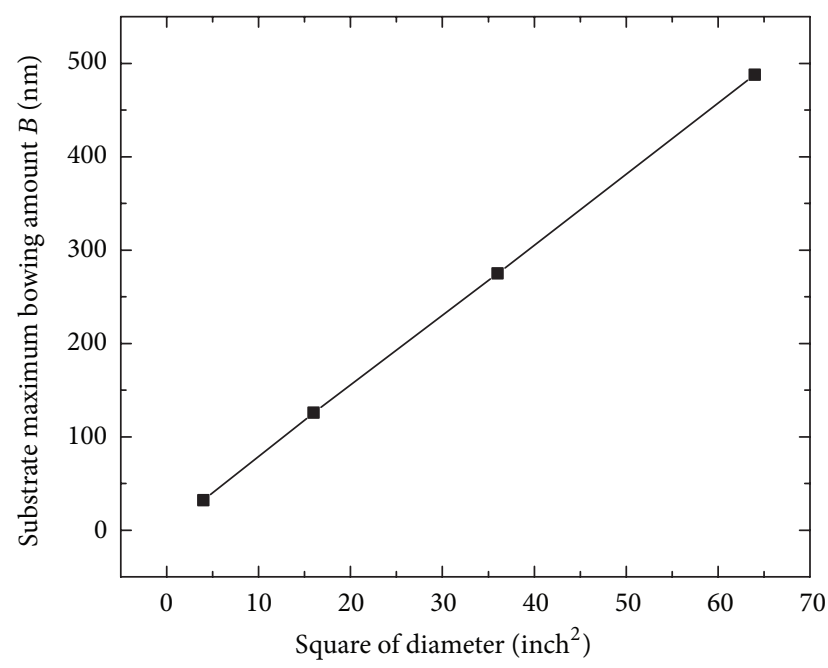

Figure 5: The linear relationship between the bowing amount and the square of sapphire diameter.

surfaces also changes. The average temperature difference between the upper and lower surfaces is calculated, and its relationship with the thickness $d$ of the substrate is shown as in Figure 7. A roughly linear relationship can be seen between the two; that is, $\Delta T / d \approx C$. The result shows that the thickness of the substrate is the main reason for the temperature difference between the upper and lower substrate surfaces, and generally there is a direct proportion linear relationship between them. Based on formula (11), to further simplify the result, we can get

$$
B \approx \frac{1}{2} \alpha R^{2} \frac{\Delta T}{d}=\frac{1}{2} \alpha R^{2} C=\left(\frac{1}{2} \alpha C\right) R^{2}=A R^{2} .
$$

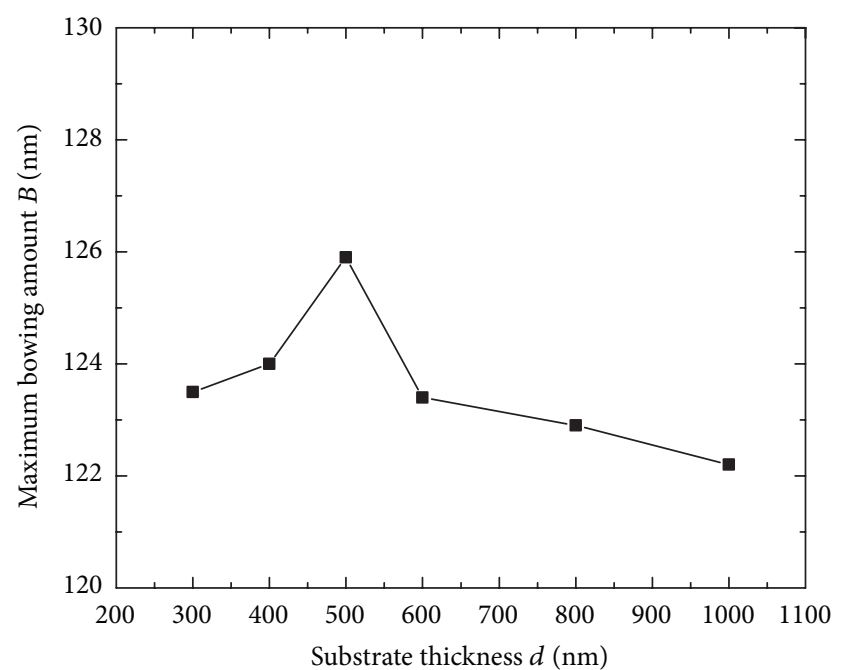

FIGURE 6: Bowing with different substrate thicknesses.

\section{Conclusions}

The bowing of epitaxial wafer is the main reason leading to plate deformation and is also one of the major difficulties in the growth of large-size epitaxial wafer; the degree of bowing can influence the uniformity of surface temperature field and flow field. In this paper, through the simulation and mathematical derivation, the bowing deformation of the $\mathrm{GaN}$ wafer on sapphire substrate is studied in detail. It is found that the epitaxial wafer bowing has a linear relationship with the square of the diameter of the substrate. Within a certain range, there is little relationship between the degree of bowing of the epitaxial wafer and its thickness. This is 


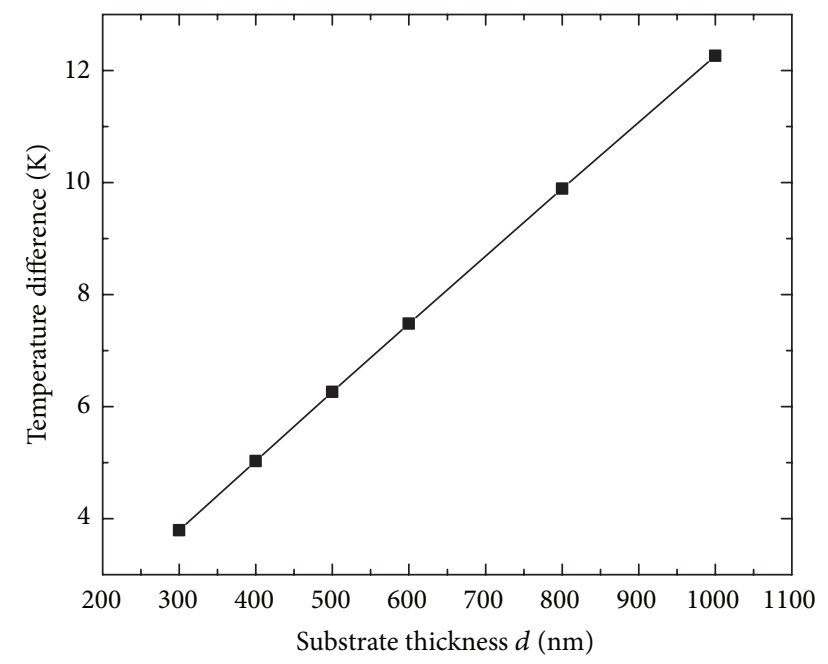

FIGURE 7: Relationship between substrate thickness and surface temperature difference.

due to the direct proportion linear relationship between the temperature difference of the upper and lower surfaces and thickness. The mathematical derivation demonstrates that the epitaxial wafer bowing amount can be represented by using the formula $B \approx(1 / 2) \alpha(\Delta T / d) R^{2}$. Under normal circumstances, $\Delta T / d$ is approximately constant.

\section{Acknowledgment}

This project is supported by the National Natural Science Foundation of China (Grant nos. 61376076, 61274077, 61274026, and 61377024); the Science and Technology Plan Foundation of Hunan Province (Grant nos. 2013FJ2011 and 2013FJ4232); the Scientific Research Fund of Hunan Provincial Education Department (Grant nos. 12C0108 and 13C321).

\section{References}

[1] J. Xue, J. Zhang, Y. Hou, H. Zhou, J. Zhang, and Y. Hao, "Pulsed metal organic chemical vapor deposition of nearly latticedmatched InAlN/GaN/InAlN/GaN double-channel high electron mobility transistors," Applied Physics Letters, vol. 100, no. 1, Article ID 013507, 2012.

[2] Y. Lianqiao, C. Zunmiao, Z. Jianhua, and A. G. Li, “Transport phenomena in a novel large MOCVD reactor for epitaxial growth of GaN thin films," IEEE Transactions on Semiconductor Manufacturing, vol. 25, no. 1, pp. 16-18, 2012.

[3] Z.-Q. Cheng, S. Hu, W.-J. Zhou, and J. Liu, "Effect of composited-layer AlyGal-yN on performances of AlGaN/GaN HEMT with unintentionally doping barrier AlxGa 1-xN," Microwave and Optical Technology Letters, vol. 53, no. 6, pp. 1206-1209, 2011.

[4] Z.-Q. Cheng, S. Hu, J. Liu, and Q.-J. Zhang, "Novel model of a AlGaN/GaN high electron mobility transistor based on an artificial neural network," Chinese Physics B, vol. 20, no. 3, Article ID 036106, 2011.

[5] H. O. Li, C. W. Tang, K. J. Chen, and K. M. Lau, "Metamorphic InAlAs/InGaAs HEMTs on GaAs substrates grown by
MOCVD," Electron Device Letters, vol. 29, no. 6, pp. 561-564, 2008.

[6] M. H. Lo, P. M. Tu, C. H. Wang et al., "High efficiency light emitting diode with anisotropically etched GaN-sapphire interface," Applied Physics Letters, vol. 95, no. 4, Article ID 041109, 2009.

[7] Y. J. Lee, J. M. Hwang, T. C. Hsu et al., "Enhancing the output power of GaN-based LEDs grown on wet-etched patterned sapphire substrates," IEEE Photonics Technology Letters, vol. 18, no. 10, pp. 1152-1154, 2006.

[8] T. Tao, Z. Zhang, L. Liu et al., "Surface morphology and composition studies in InGaN/GaN film grown by MOCVD," Journal of Semiconductors, vol. 32, no. 8, Article ID 083002, 2011.

[9] Y. Wang, J. Li, S. Li, H. Chen, D. Liu, and J. Kang, "X-ray reflectivity and atomic force microscopy studies of MOCVD grown AlxGal-xN/GaN superlattice structures," Journal of Semiconductors, vol. 32, no. 4, Article ID 043006, 2011.

[10] Y. Liu, H. Chen, and S. Fu, "CFD simulation of flow patterns in GaN-MOCVD reactor," Chinese Journal of Semiconductors, vol. 25, no. 12, pp. 1639-1646, 2004.

[11] Z. M. Li, S. R. Xu, J. C. Zhang et al., "Finite Element Analysis of the Temperature Field in a Vertical MOCVD Reactor by Induction Heating," Journal of Semiconductors, vol. 33, no. 11, Article ID 113004, 5 pages, 2009.

[12] Z. M. Li, Y. Hao, J. C. Zhang et al., “Thermal transportation simulation of a susceptor structure with ring groove for the vertical MOCVD reactor," Journal of Crystal Growth, vol. 311, no. 23-24, pp. 4679-4684, 2009.

[13] H. Ying-lu, L. Pei-xian, L. Zhi-ming et al., "Simulation and analysis of temperature modulate curve in MOCVD with the chipped infrared heating system," Electronic Science and Technology, vol. 25, no. 1, pp. 108-111, 2012.

[14] S.-Q. Zhong, X.-M. Ren, Q. Wang et al., "Numerical simulation of flow and temperature field in MOCVD reactor," Journal of Synthetic Crystals, vol. 37, no. 6, pp. 1342-1348, 2008.

[15] S. R. Xu, Y. Hao, J. C. Zhang et al., "Yellow luminescence of polar and nonpolar gan nanowires on $r$-plane sapphire by metal organic chemical vapor deposition," Nano Letters, vol. 13, pp. 3654-3657, 2013.

[16] Q. Xu, R. Zuo, and H. Zhang, "Design and simulation of reverseflow showerhead MOCVD reactors," Journal of Synthetic Crystals, vol. 34, no. 6, pp. 1059-1064, 2005.

[17] G.-B. Wang, R. Zuo, Q. Xu, H. Li, H.-Q. Yu, and J.-S. Chen, "Design and numerical simulation of MOCVD reactor with tangential inlets," Journal of Synthetic Crystals, vol. 39, no. 1, pp. 267-271, 2010. 

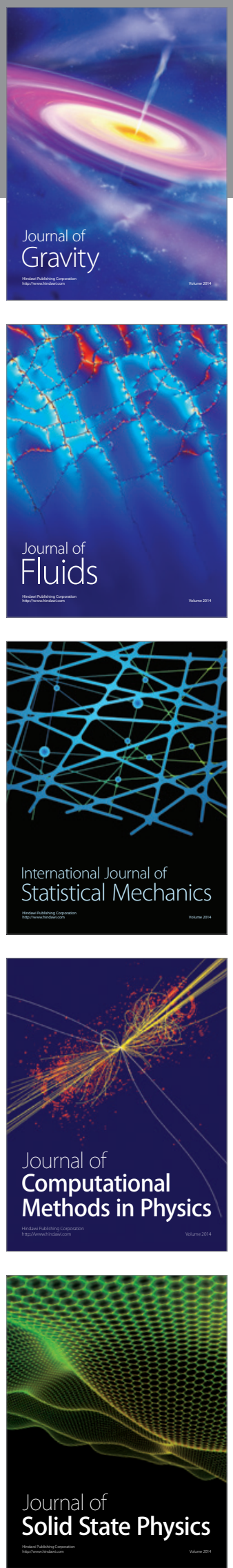

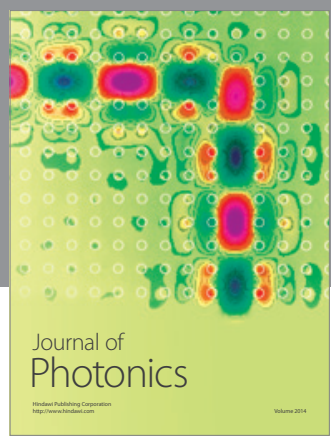

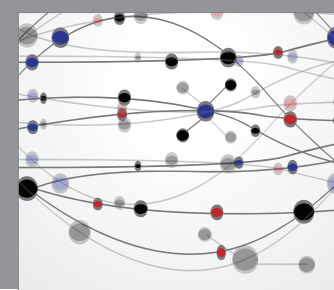

The Scientific World Journal

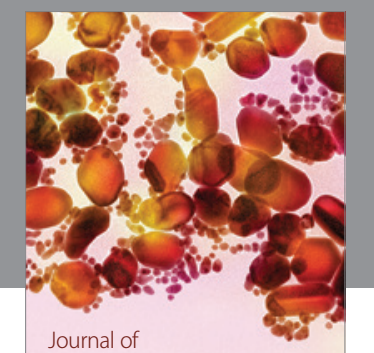

Soft Matter
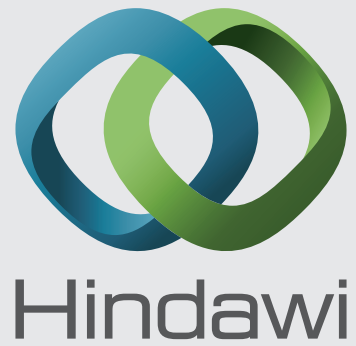

Submit your manuscripts at

http://www.hindawi.com
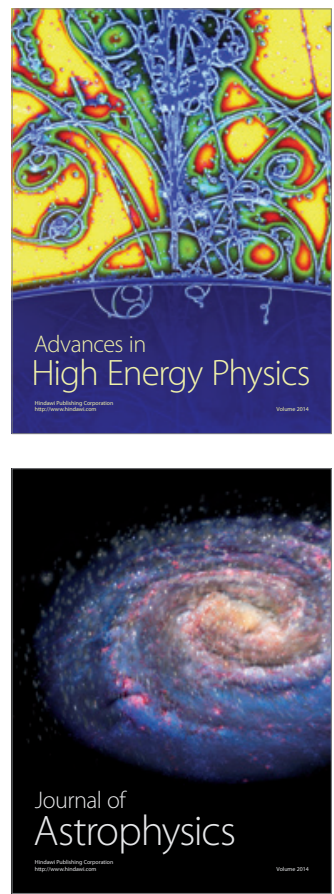
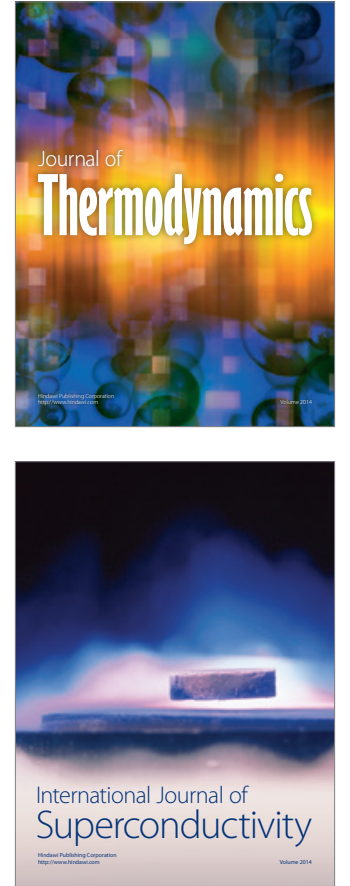
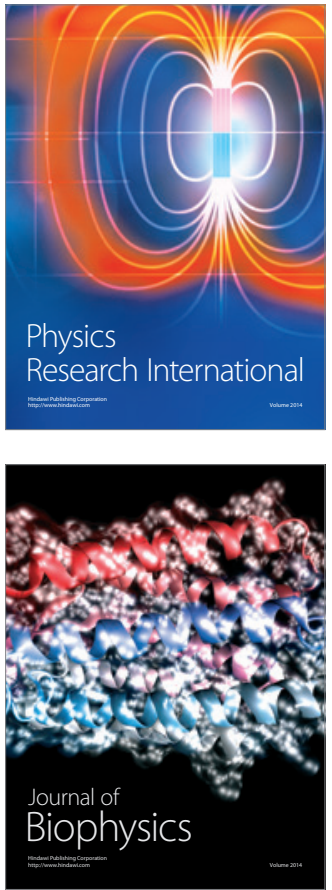
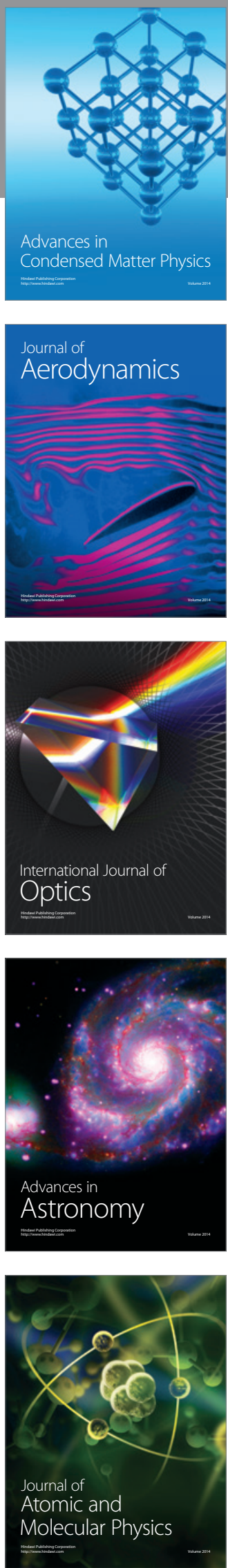